\title{
Tissue inhibitor of metalloproteinases 1, a novel biomarker of tuberculosis
}

\author{
YINGYU CHEN $^{1-3}$, JIERU WANG $^{1,2}$, PAN GE $^{1,2}$, DEJUN CAO $^{1,2}$, BEIPING MIAO $^{1,2}$, \\ IAN ROBERTSON ${ }^{3,4}$, XIA ZHOU $^{5}$, LI ZHANG $^{5}$, HUANCHUN CHEN ${ }^{1,5}$ and AIZHEN GUO ${ }^{1-3}$
}

\author{
${ }^{1}$ The State Key Laboratory of Agricultural Microbiology, Huazhong Agricultural University; ${ }^{2}$ College of Veterinary Medicine, \\ Huazhong Agricultural University; ${ }^{3}$ China Australia Joint Research and Training Center for Veterinary Epidemiology, \\ Wuhan, Hubei 430070, P.R. China; ${ }^{4}$ School of Veterinary and Life Sciences, College of Veterinary Medicine, \\ Murdoch University, Murdoch, Western Australia 6150, Australia; ${ }^{5}$ Tuberculosis Department, \\ Wuhan Medical Treatment Center, Wuhan, Hubei 430023, P.R. China
}

Received May 17, 2016; Accepted October 24, 2016

DOI: $10.3892 / \mathrm{mmr} .2016 .5998$

\begin{abstract}
Tuberculosis (TB) is an important infectious disease of humans and other animals. Conventional diagnostic methods, including the tuberculin skin test, chest X-rays and bacterial culture, have certain innate disadvantages for the early, rapid and specific diagnosis of tuberculosis. The present study aimed to identify a novel diagnostic biomarker to overcome these disadvantages. The potential target identified in the present study was tissue inhibitor of metalloproteinases 1 (TIMP-1), which has previously been demonstrated to be critical in the immune response to TB. The concentration of TIMP-1 in the blood was determined using a commercial ELISA kit, and the relative mRNA expression levels following bacterial infection were detected by reverse transcription-quantitative polymerase chain reaction. Based on a clinical and microbiological diagnosis, the ELISA for plasma TIMP-1 had a sensitivity of $91.80 \%$ [95\% confidence interval (CI): $85.44,96.00]$ and a specificity of $91.41 \%$ (95\% CI: 85.14, 95.63). In a THP-1 cell model, Bacillus Calmette-Guérin and Mycobacterium bovis significantly upregulated the mRNA expression levels of TIMP-1 post infection in a time-dependent manner $(\mathrm{P}=0.006$ for BCG 24 h PI, $\mathrm{P}=3.2 \times 10^{-7}$ for $M$. bovis $24 \mathrm{PI}$ ). The results of the present study indicate that plasma TIMP-1 may be a potential biomarker for the diagnosis of TB.
\end{abstract}

Correspondence to: Dr Aizhen Guo, College of Veterinary Medicine, Huazhong Agricultural University, 1 Shizishan Street, Hongshan, Wuhan, Hubei 430070, P.R. China

E-mail: aizhen@mail.hzau.edu.cn

Key words: tuberculosis, tissue inhibitor of metalloproteinases 1, diagnosis, biomarker

\section{Introduction}

Tuberculosis (TB), caused by Mycobacterium tuberculosis $(M . t b)$, is one of the most important infectious diseases of humans, with an estimated 9 million new cases and 1.5 million fatalities worldwide in 2013 (1). The treatment of TB requires a six-month program; the treatment for drug-resistant TB is longer (at least 18 months) and requires the use of more toxic drugs (2). Consequently, early and accurate diagnosis of TB may potentially increase treatment efficacy and reduce patient suffering. However, conventional diagnostic methods, including culture and microscopic examination of sputa, the tuberculin skin test (TST), chest $\mathrm{X}$-ray, bronchial endoscopy and polymerase chain reaction (PCR) typically produce high proportions of false negative and positive results $(3,4)$. Blood-based laboratory tests, including the interferon- $\gamma(\mathrm{IFN}-\gamma)$ in vitro release assay and antibody detection, have greater sensitivity and specificity compared with the conventional diagnostic methods; however, they do not differentiate between latent and active infections (5). Therefore, novel diagnostic biomarkers with high sensitivity and specificity are urgently required to accurately diagnose $\mathrm{TB}$, and to differentiate between the different forms of TB (6).

Recently, tissue inhibitors of metalloproteinases (TIMPs) have been suggested as potential biomarkers for TB $(7,8)$. TIMPs (TIMP-1, -2 and -3) facilitate the remodeling and repair of tissue following destruction by matrix metalloproteinases (MMPs). For example, the concentrations of MMP-1, -3 and -8 have been demonstrated to decrease rapidly during TB treatment, in contrast to transient increases in the concentrations of TIMP-1 and -2 at week 2 of treatment (7). In addition, TIMP-1 has been revealed to be responsible for residual pleural thickening in pleural tuberculosis (9).

Our previous investigations to identify potential TB biomarkers in the sera of patients using protein microarrays demonstrated that TIMP-1 is a primary component associated with the occurrence of disease (unpublished data). However, the role of TIMP-1 in TB diagnosis has rarely been studied. Therefore, the present study aimed to evaluate the association 
of TIMP-1 with TB, and to investigate the potential of TIMP-1 as a biomarker to aid in the diagnosis of TB.

\section{Materials and methods}

Subjects. A total of 122 patients who were confirmed to have active TB based upon presenting clinical symptoms and/or culture and/or chest X-ray at the Tuberculosis Department, Wuhan Medical Treatment Center (Wuhan, China), were recruited. A further 37 pneumonia patients were enrolled at Zhongnan Hospital (Wuhan, China). In addition, 128 healthy volunteers from Huazhong Agricultural University (Wuhan, China), who had a negative TST, a negative chest X-ray and had no known exposure to TB, were included in the present study as a control group. All subjects tested negative for human immunodeficiency virus. Informed consent was obtained from all participants. The present study was approved by the Research Ethics Committee of Huazhong Agricultural University.

Blood collection. Heparinized venous blood $(5 \mathrm{ml})$ was collected from the antecubital vein of all subjects. For each sample, plasma was isolated and stored at $-20^{\circ} \mathrm{C}$ for the subsequent detection of TIMP-1. Peripheral blood mononuclear cells (PBMCs) were isolated from blood samples and stimulated with CFP-10/ESAT-6 (stocked in our own lab), or mock-stimulated with PBS, overnight (16 h) as described previously (10).

Measurement of TIMP-1 concentrations. Commercial ELISA kits for TIMP-1 (RayBiotech, Inc., Norcross, GA, USA, cat. no. ELH-TIMP1) were used according to the manufacturer's protocol, to analyze the levels of TIMP-1 in plasma. Briefly, $100 \mu \mathrm{l}$ of each sample was added to each well of an ELISA plate. The plates were incubated for $2.5 \mathrm{~h}$ at room temperature and washed. A biotin-conjugated antibody (100 $\mu \mathrm{l})$ was added to each well and the plates incubated for a further $1 \mathrm{~h}$ at room temperature. Following washing, $100 \mu$ l horseradish peroxidase-streptavidin solution was added to each well and the plate was incubated at room temperature for $45 \mathrm{~min}$. The color was developed with the substrate 3,3',5,5'-tetramethylbenzidine $/ \mathrm{H}_{2} \mathrm{O}_{2}$ for $30 \mathrm{~min}$ at room temperature, and the reaction was terminated by adding the stop solution. The optical density was measured at a wavelength of $450 \mathrm{~nm}$ using a microplate reader and the concentrations were calculated based on the standard curve.

Bacterial culture. Mycobacterium bovis [M. bovis; American Type Culture Collection (ATCC) 19210] and Bacillus Calmette-Guérin (BCG) Tokyo strain (ATCC 35737) were donated by Dr Chuan-You Li (Beijing Tuberculosis \& Thoracic Tumor Research Institute, Beijing, China). The two strains were cultured in $250 \mathrm{ml}$ Middlebrook 7H9 broth (BD Biosciences, Franklin Lakes, NJ, USA) supplemented with $10 \%$ oleic acid-albumin-dextrose-catalase (BD Biosciences) and 0.05\% Tween 80 (Amresco, LLC, Solon, OH, USA), with shaking in the Biosafey Level 3 facility at Huazhong Agricultural University. Following incubation at $37^{\circ} \mathrm{C}$ for 7 days until growth reached the log phase, the bacilli were harvested from the media and centrifuged at 5,000 x $\mathrm{g}$ for $10 \mathrm{~min}$ at room temperature. The pellets were transferred into a mortar grinder and homogenized, and subsequently resuspended in $40 \mathrm{ml}$
Middlebrook $7 \mathrm{H} 9$ broth. Following a 5-min incubation, the supernatant was collected, mixed, aliquoted into $1 \mathrm{ml}$ tubes and stored at $-80^{\circ} \mathrm{C}$ until further use. The middle tube was taken and the number of colony forming units was determined via a dilution-plating assay, as previously described (11).

Analysis of TIMP-1 mRNA expression levels. The THP-1 human monocytic cell line was provided by Dr Chuan-You Li (Beijing Tuberculosis \& Thoracic Tumor Research Institute, Beijing, China) and was prepared and the infection procedures were conducted as previously described (12). Briefly, the bacteria (M. bovis and BCG) were added to 24-well cell culture plates and cultured for $24 \mathrm{~h}$ at a multiplicity of infection rate of 10. Total RNA was extracted as described previously (12), and subjected to reverse transcription-quantitative PCR (RT-qPCR). RNA was reverse-transcribed using a Reverse Transcription kit (Toyobo Co., Ltd., Osaka, Japan). During the RNA isolation and reverse transcription, RNase-free reagents and consumables were used. Real-time quantitative PCR (qPCR) was performed using THUNDERBIRD SYBR qPCR mix (Toyobo Co., Ltd., Osaka, Japan). The volume of each reaction was $25 \mu \mathrm{l}$, including $100 \mathrm{ng}$ cDNA, $200 \mathrm{nmol}$ of each primer and $12.5 \mu \mathrm{l} 2 \mathrm{xSYBR}-\mathrm{Green}$ dye. Reactions were programmed in Roche LightCycler ${ }^{\circledR} 480$ (Roche Diagnostics, Basel, Switzerland) as follows: $95^{\circ} \mathrm{C}$ for $10 \mathrm{~min}$, followed by 30 cycles of $95^{\circ} \mathrm{Cfor} 30 \mathrm{sec}, 58^{\circ} \mathrm{C}$ for $30 \mathrm{sec}$ and $72^{\circ} \mathrm{C}$ for $45 \mathrm{sec}$. The fluorescence signal was detected at the end of each elongation step. Primers (presented in Table I) were designed and commercially synthesized by the Beijing Genomics Institute (Beijing, China) for RT-qPCR to determine the mRNA expression levels of TIMP-1 in BCG- and $M$. bovis-infected THP-1 cells; $\mu$-actin served as the internal reference, the relative expression levels were quantified using the $2^{-\Delta \Delta \mathrm{Cq}}$ method (13).

Statistical analysis. Data were analyzed using a Student's $t$-test or analysis of variance. $\mathrm{P}<0.05$ was considered to indicate a statistically significant difference. Cut-off values and corresponding test sensitivity and specificity were calculated through receiver operating characteristic (ROC) curve analysis and assessing the area under the curve using Microsoft Excel software, version 2013 (Microsoft Corporation, Redmond, WA, USA), as previously described (14).

\section{Results}

TB patients have increased serum levels of TIMP-1 compared with healthy controls and pneumonia patients. The baseline serum TIMP-1 levels of patients with tuberculosis were significantly greater compared with the pneumonia $(\mathrm{P}=0.02)$ and healthy control groups $\left(\mathrm{P}=8 \times 10^{-4}\right)$, with median values of 1201, 1140 and $415.4 \mathrm{ng} / \mathrm{ml}$, respectively (Fig. 1). In addition TIMP-1 levels in pneumonia patients were significantly greater compared with healthy controls $\left(\mathrm{P}=2 \times 10^{-11}\right)$.

According to the ROC, a cut-off value of $727 \mathrm{ng} / \mathrm{ml}$ was set to maximize discrimination between positive and negative results for TIMP-1 in the TB patients group. TIMP-1 levels were significantly greater in $112 \mathrm{~TB}$ patients [mean \pm standard deviation (SD), $1348 \pm 607.3 \mathrm{ng} / \mathrm{ml}$ ] compared with healthy controls (mean $\pm \mathrm{SD}, 400.4 \pm 292.2 \mathrm{ng} / \mathrm{ml}$; $\mathrm{P}<0.0001$ ). At this 
Table I. Primers for reverse transcription-quantitative polymerase chain reaction.

\begin{tabular}{|c|c|c|c|c|}
\hline Gene & Direction & Sequence $\left(5^{\prime}-3^{\prime}\right)$ & Product size (bp) & GenBank accession no. \\
\hline \multirow[t]{2}{*}{$\beta$-actin } & $\mathrm{F}$ & AGCGAGCATCCCCCAAAGTT & 285 & ВC002409 \\
\hline & $\mathrm{R}$ & GGGCACGAAGGCTCATCATT & & \\
\hline \multirow[t]{2}{*}{ TIMP-1 } & $\mathrm{F}$ & CTGCGGATACTTCCACAGGTC & 168 & NM_003254 \\
\hline & $\mathrm{R}$ & TTCTGGATGTGACAACCGACAACCGACACT & & \\
\hline
\end{tabular}

F, forward; R, reverse; TIMP-1, tuberculosis, tissue inhibitor of metalloproteinases 1.

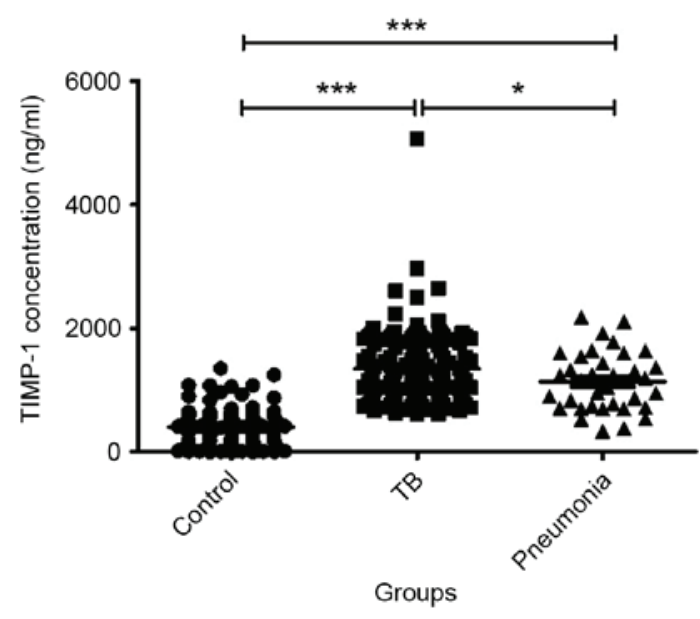

Figure 1. TIMP-1 concentration in sera. TIMP-1 levels in the sera of TB and pneumonia patients and healthy controls were detected using a commercial ELISA kit. TIMP-1 levels were significantly increased in TB patients compared with pneumonia and healthy control groups. TIMP-1, tissue inhibitor of metalloproteinases 1 ; TB, tuberculosis. ${ }^{*} \mathrm{P}<0.05,{ }^{* *} \mathrm{P}<0.01,{ }^{* * *} \mathrm{P}<0.001$.

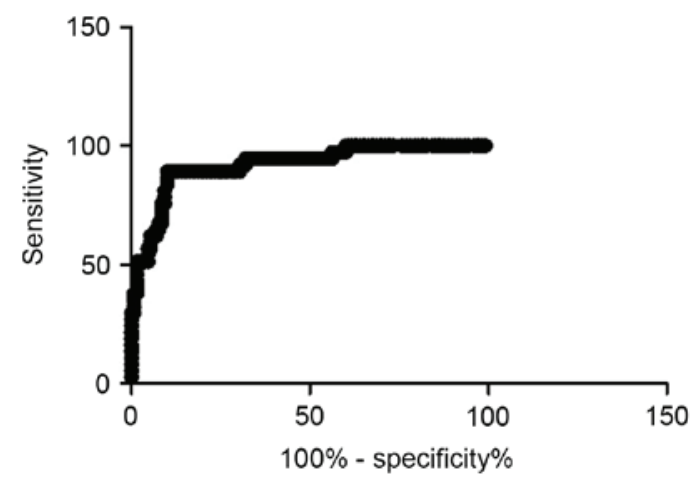

Figure 2. Receiver operating characteristic curve for serum TIMP-1 levels between TB patients and healthy controls. A cut-off value of $727 \mathrm{ng} / \mathrm{ml}$ was set. Based on clinical diagnosis, the TIMP-1 ELISA had a sensitivity of $91.80 \%$ and a specificity of $91.41 \%$. TIMP-1, tissue inhibitor of metalloproteinases 1; TB, tuberculosis.

cut-off point, $91.80 \%$ [112/122; 95\% confidence interval (CI): $85.44,96.00]$ of TB patients were classified as test-positive compared with only $8.59 \%(11 / 128 ; 95 \%$ CI: $4.37,14.86)$ of healthy controls. Using clinical diagnosis as the gold standard, the TIMP-1 ELISA had a sensitivity of $91.80 \%$ (95\% CI: 85.44 , 96.00) and a specificity of $91.41 \%$ (95\% CI: 85.14, 95.63; Fig. 2).

Furthermore, $1037 \mathrm{ng} / \mathrm{ml}$ was set as the cut-off point to distinguish TB and pneumonia patients according to ROC,

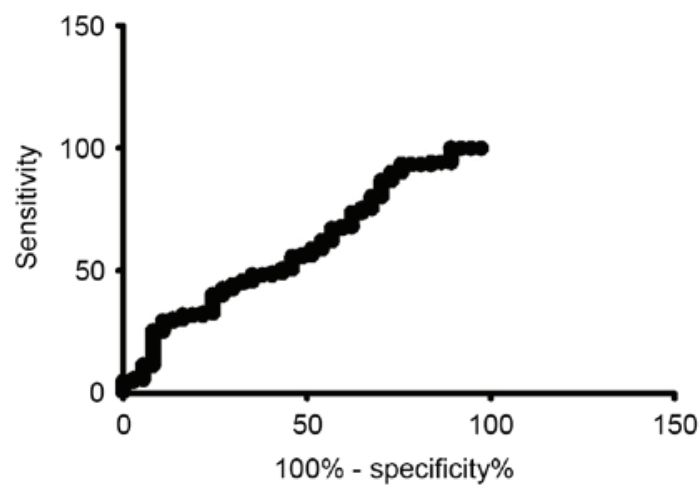

Figure 3. Receiver operating characteristic curve for serum TIMP-1 levels between TB patients and pneumonia patients. A cut-off value of $1037 \mathrm{ng} / \mathrm{ml}$ was set. The TIMP-1 ELISA had a sensitivity of $62.3 \%$ and a specificity of $45.95 \%$. TIMP-1, tissue inhibitor of metalloproteinases 1; TB, tuberculosis.

with a sensitivity of $62.3 \%$ (95\% CI: 53.07, 70.91), and a specificity of $45.95 \%$ (95\% CI: 29.49, 63.08; Fig. 3).

TIMP-1 production by PBMC following stimulation with CFP-10/ESAT-6. PBMCs isolated from the blood of 38 TB patients and 38 healthy controls were stimulated with CFP-10/ESAT- 6 or mock-stimulated with PBS. CFP-10/ESAT-6 did not induce PBMCs to produce TIMP-1 in TB patients $(\mathrm{P}=0.3051)$. Similarly, there was no difference in TIMP-1 levels between healthy control PBMCs incubated with CFP-10/ESAT-6 or PBS ( $\mathrm{P}=0.1158)$. The TB samples treated with CFP-10/ESAT-6 or PBS had significantly greater TIMP-1 levels compared with healthy controls ( $\mathrm{P}<0.0001$; Fig. 4).

mRNA expression levels of TIMP-1 following infection with $B C G$ or $M$. bovis. TIMP-1 mRNA expression levels were detected by RT-qPCR at 12 and $24 \mathrm{~h}$ post infection and normalized to $\mu$-actin mRNA expression levels. TIMP-1 mRNA expression levels were significantly upregulated in a time-dependent manner following BCG and M. bovis infection. BCG and $M$. bovis infection significantly increased TIMP-1 mRNA expression levels at $24 \mathrm{~h}$ post infection $(\mathrm{P}=0.006$ for BCG 24 h PI; $\mathrm{P}=3.2 \times 10^{-7}$ for M.bovis $24 \mathrm{PI}$; Fig. 5).

\section{Discussion}

Tuberculosis has been recognized in humans for centuries and is a potentially life-threatening or debilitating disease. Advances in the diagnosis of the disease may result in control 


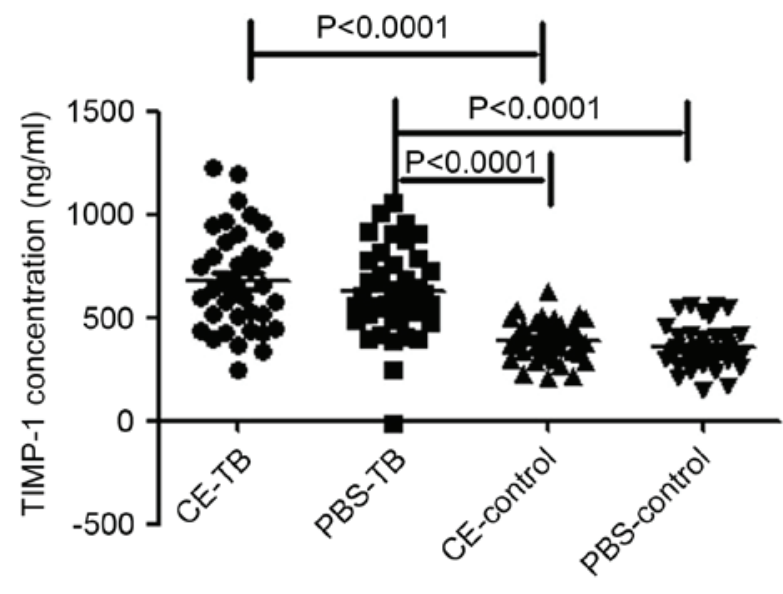

Stimulation groups

Figure 4. TIMP-1 levels in plasma following stimulation. PBMCs isolated from the blood of TB patients and healthy controls were stimulated with CFP-10/ESAT-6 or mock-stimulated with PBS. The plasma TIMP-1 concentrations were subsequently measured using a commercial ELISA kit. Stimulation with CFP-10/ESAT-6 did not induce PBMCs from TB patients or healthy controls to produce TIMP-1. TB PBMCs had greater TIMP-1 levels compared with PBMCs from healthy controls. TIMP-1, tissue inhibitor of metalloproteinases 1; TB, tuberculosis; PBMCs, peripheral blood mononuclear cells; CE, CFP-10/ESAT-6.

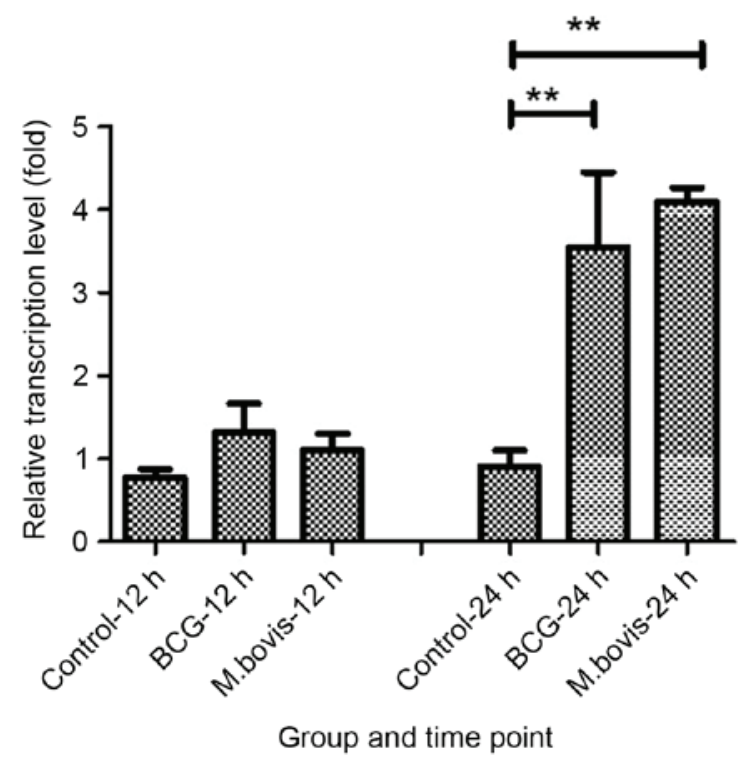

Figure 5. TIMP-1 mRNA expression levels in the THP-1 human monocytic cell line following infection with BCG or M. bovis. TIMP-1 mRNA expression levels were significantly upregulated in a time-dependent manner following BCG and $M$. bovis infection. TIMP-1, tissue inhibitor of metalloproteinases 1; TB, tuberculosis; BCG, Bacillus Calmette-Guérin; M. bovis, Mycobacterium bovis. ${ }^{* *} \mathrm{P}<0.01$.

measures being implemented more rapidly (15), reducing the impact of the disease on the community. In the present study the plasma TIMP-1 ELISA was revealed to have a sensitivity of $91.80 \%$ (95\% CI: $85.44,96.00)$ and a specificity of $91.41 \%$ (95\% CI: 85.14, 95.63) according to ROC.

MMPs are involved in TB in the migration of leukocytes to infection sites and tissue destruction (16). Cytokines, including tumor necrosis factor- $\alpha$ and IFN- $\gamma$, which may be induced by $M . t b$ infection, upregulate MMP production in recruited monocytes and macrophages (8). TIMP-1 is an inhibitor of MMPs, and controls MMP activity by forming 1:1 complexes with MMPs, regulating the proteolysis of connective tissues and controlling tissue damage (17). Previous studies have demonstrated that concentrations of MMP-1, $-2,-3,-8$ and -9 , as well as TIMP-1/2, are significantly greater in TB patients compared with healthy controls $(7,18-20)$. In the present study, serum TIMP-1 levels were significantly increased in TB patients compared with healthy controls, similar to previous studies $(9,18,19)$. As TIMP production is associated with tissue destruction, tests for TIMP levels have the potential to differentiate active TB from latent infection. This represents a potential advantage over the commonly used IFN- $\gamma$ in vitro release assay, which is based on cellular immunity memory to $M$. $t b$ infection and which does not differentiate active TB from latent infection (21).

To support this hypothesis indirectly, the present study used the $M$. $t b$-specific antigen CFP-10/ESAT- 6 to stimulate PBMCs. However, CFP-10/ESAT-6 stimulation did not significantly alter the plasma TIMP-1 concentrations in TB patients. These results indicated that TIMP-1 was not produced by PBMCs following $M . t b$ antigen stimulation, and was affected by the tissue damage induced by virulent bacteria or BCG. However, this finding requires confirmation in a larger cohort.

In conclusion, the present study demonstrated that TIMP-1 is present at high levels in TB patient sera, and that expression of TIMP-1 mRNA is induced by mycobacteria. TIMP-1 may therefore be a potential biomarker of TB in humans.

\section{Acknowledgements}

The present study was supported by the Key Special Science and Technology Program for Important Infectious Diseases Such as AIDS and Viral Hepatitis (grant nos. 2012ZX10003 and 2012ZX10004214), the China National Basic Research (973) Program (grant no. 2012CB518801), the National Natural Science Foundation of China (grant no. 31421064) and the Fok Ying Tung Education Foundation (grant no. 132026).

\section{References}

1. World Health Organisation (WHO): Global tuberculosis report 2014.

2. Qadeer E, Fatima R, Fielding K, Qazi F, Moore D and Khan MS: Good quality locally procured drugs can be as effective as internationally quality assured drugs in treating multi-drug resistant tuberculosis. PLoS One 10: e0126099, 2015.

3. Tiwari D, Tiwari RP, Chandra R, Bisen PS and Haque S: Efficient ELISA for Diagnosis of Active Tuberculosis Employing a Cocktail of Secretory Proteins of Mycobacterium tuberculosis. Folia Biol (Praha) 60: 10-20, 2014.

4. Hajiabdolbaghi M, Rasoulinejad M, Davoudi AR, Alikhani A and Najafi N: Application of peripheral blood Mycobacterium tuberculosis PCR for diagnosis of tuberculosis patients. Eur Rev Med Pharmacol Sci 18: 185-189, 2014.

5. Chen Y, Deng Q, Zhan Z, Guo A, Xiang J, Chen J, Zhou J, Zeng Q, Wei W, Tong Q, et al: Establishment of human IFN-gamma in vitro release assay and its application in tuberculosis diagnosis. Sheng Wu Gong Cheng Xue Bao 24: 1653-1657, 2008 (In Chinese).

6. Bwanga F, Hoffner S, Haile M and Joloba ML: Direct susceptibility testing for multi drug resistant tuberculosis: A meta-analysis. BMC Infect Dis 9: 67, 2009. 
7. Ugarte-Gil CA, Elkington P, Gilman RH, Coronel J, Tezera LB, Bernabe-Ortiz A, Gotuzzo E, Friedland JS and Moore DA: Induced sputum MMP-1, -3 \& -8 concentrations during treatment of tuberculosis. PLoS One 8: e61333, 2013.

8. Sundararajan S, Babu S and Das SD: Comparison of localized versus systemic levels of Matrix metalloproteinases (MMPs), its tissue inhibitors (TIMPs) and cytokines in tuberculous and non-tuberculous pleuritis patients. Hum Immunol 73: 985-991, 2012.

9. Hwang KE, Shon YJ, Cha BK, Park MJ, Chu MS, Kim YJ, Jeong ET and Kim HR: Tissue inhibitor of metalloproteinase-1 is responsible for residual pleural thickening in pleural tuberculosis. Tohoku J Exp Med 235: 327-333, 2015.

10. Chen Y, Chao Y, Deng Q, Liu T, Xiang J, Chen J, Zhou J, Zhan Z, Kuang Y, Cai H, et al: Potential challenges to the Stop TB Plan for humans in China; cattle maintain M. bovis and $\mathrm{M}$. tuberculosis. Tuberculosis (Edinb) 89: 95-100, 2009.

11. Zhang X, Li S, Luo Y, Chen Y, Cheng S, Zhang G, Hu C, Chen H and Guo A: Mycobacterium bovis and BCG induce different patterns of cytokine and chemokine production in dendritic cells and differentiation patterns in CD4+ T cells. Microbiology 159: 366-379, 2013.

12. Fontán P, Aris V, Ghanny S, Soteropoulos P and Smith I: Global transcriptional profile of Mycobacterium tuberculosis during THP-1 human macrophage infection. Infect Immun 76: 717-725, 2008.

13. Weglarz L, Molin I, Orchel A, Parfiniewicz B and Dzierzewicz Z: Quantitative analysis of the level of p53 and p21(WAF1) mRNA in human colon cancer HT-29 cells treated with inositol hexaphosphate. Acta Biochim Pol 53:349-356, 2006.

14. Gall D and Nielsen K: Comparison of some methods for determing cutoff values for serological assays: A retrospective study using the fluorescence polarization assay. J Immunoassay Immunochem 22: 85-98, 2001.
15. Small PM and Pai M: Tuberculosis diagnosis-time for a game change. N Engl J Med 363: 1070-1071, 2010.

16. Rand L, Green JA, Saraiva L, Friedland JS and Elkington PT: Matrix metalloproteinase-1 is regulated in tuberculosis by a p38 MAPK-dependent, $\mathrm{p}$-aminosalicylic acid-sensitive signaling cascade. J Immunol 182: 5865-5872, 2009.

17. Anand SP and Selvaraj P: Effect of 1,25 dihydroxyvitamin D(3) on matrix metalloproteinases MMP-7, MMP-9 and the inhibitor TIMP-1 in pulmonary tuberculosis. Clin Immunol 133: 126-131, 2009.

18. Elkington P, Shiomi T, Breen R, Nuttall RK, Ugarte-Gil CA, Walker NF, Saraiva L, Pedersen B, Mauri F, Lipman M, et al: MMP-1 drives immunopathology in human tuberculosis and transgenic mice. J Clin Invest 121: 1827-1833, 2011.

19. Walker NF, Clark SO, Oni T, Andreu N, Tezera L, Singh S, Saraiva L, Pedersen B, Kelly DL, Tree JA, et al: Doxycycline and HIV infection suppress tuberculosis-induced matrix metalloproteinases. Am J Respir Crit Care Med 185: 989-997, 2012.

20. Hoheisel G, Sack U, Hui DS, Huse K, Chan KS, Chan KK, Hartwig K, Schuster E, Scholz GH and Schauer J: Occurrence of matrix metalloproteinases and tissue inhibitors of metalloproteinases in tuberculous pleuritis. Tuberculosis (Edinb) 81: 203-209, 2001

21. Tincati C, Cappione Iii AJ and Snyder-Cappione JE: Distinguishing Latent from Active Mycobacterium tuberculosis Infection Using Elispot Assays: Looking Beyond Interferon-gamma. Cells 1: 89-99, 2012. 